\title{
Blood Volume Analysis: A New Technique and New Clinical Interest Reinvigorate a Classic Study*
}

\author{
Timothy A. Manzone ${ }^{1}$, Hung Q. Dam ${ }^{1,2}$, Daniel Soltis ${ }^{3}$, and Vidya V. Sagar ${ }^{1}$ \\ ${ }^{I}$ Department of Nuclear Medicine, Christiana Care Health System, Newark, Delaware; ${ }^{2}$ Nuclear Medicine Section, Wilmington Veterans \\ Administration, Medical Center, Wilmington, Delaware; and ${ }^{3}$ Daxor Corporation, New York, New York
}

\begin{abstract}
Blood volume studies using the indicator dilution technique and radioactive tracers have been performed in nuclear medicine departments for over $50 \mathrm{y}$. A nuclear medicine study is the gold standard for blood volume measurement, but the classic dualisotope blood volume study is time-consuming and can be prone to technical errors. Moreover, a lack of normal values and a rubric for interpretation made volume status measurement of limited interest to most clinicians other than some hematologists. A new semiautomated system for blood volume analysis is now available and provides highly accurate results for blood volume analysis within only $90 \mathrm{~min}$. The availability of rapid, accurate blood volume analysis has brought about a surge of clinical interest in using blood volume data for clinical management. Blood volume analysis, long a low-volume nuclear medicine study all but abandoned in some laboratories, is poised to enter the clinical mainstream. This article will first present the fundamental principles of fluid balance and the clinical means of volume status assessment. We will then review the indicator dilution technique and how it is used in nuclear medicine blood volume studies. We will present an overview of the new semiautomated blood volume analysis technique, showing how the study is done, how it works, what results are provided, and how those results are interpreted. Finally, we will look at some of the emerging areas in which data from blood volume analysis can improve patient care. The reader will gain an understanding of the principles underlying blood volume assessment, know how current nuclear medicine blood volume analysis studies are performed, and appreciate their potential clinical impact.
\end{abstract}

Key Words: blood volume determination; plasma volume; erythrocyte volume; indicator dilution technique; capillary fragility

J Nucl Med Technol 2007; 35:55-63

DOI: 10.2967/jnmt.106.035972

\section{H}

ow much blood does a human have? The usual answer, "about 5-6 quarts," is obviously just a rough estimate. Of the myriad laboratory tests performed on blood every day, the results are almost always given as a con-

\footnotetext{
Received Aug. 24, 2006; revision accepted Feb. 28, 2007.

For correspondence or reprints contact: Timothy A. Manzone, MD, JD, Department of Nuclear Medicine, Christiana Care Health System, 4755

Ogletown-Stanton Rd., Newark, DE 19718.

E-mail: tmanzone@christianacare.org

*NOTE: FOR CE CREDIT, YOU CAN ACCESS THIS ACTIVITY THROUGH

THE SNM WEB SITE (http://www.snm.org/ce_online) THROUGH JUNE 2009.

COPYRIGHT ( 2007 by the Society of Nuclear Medicine, Inc.
}

centration, that is, in terms of blood volume (e.g., ng/dL, $\mathrm{mg} / \mathrm{mL}$, cells $/ \mathrm{mm}^{3}$ ) How can blood volume actually be measured in a living patient? This is an important question because the volume of circulating blood is a crucial clinical parameter. Fluid balance and circulation are complex, extremely dynamic processes. Effective perfusion and blood pressure depend on a complex interplay between cardiac output, circulating blood volume, and vasomotor tone.

Much of the art of clinical medicine consists of estimating the volume status of patients. Discussions about whether patients are "wet" or "dry" are constantly heard during hospital rounds. The wet patients are often treated with diuretics, dialysis, or fluid restriction, whereas the dry ones receive intravenous fluids or blood transfusions. The clinical assessment of volume status relies mostly on indirect indicators such as blood pressure, lung sounds, edema, jugular vein distension, changes in weight, input/output monitoring, and blood urea nitrogen/creatinine. Sometimes physicians must use a fluid challenge or invasive central pressure monitoring to assess volume status in a difficult patient.

Nuclear medicine techniques to analyze blood volume have been used for decades; however, blood volume studies tended to be time-consuming, complicated, and prone to errors. Furthermore, there were no accepted reference values for blood volume parameters. As a result of all these factors, blood volume studies were seldom used by clinicians and were discontinued by some nuclear medicine departments because of low volume and lack of interest.

Within the last several years, a new semiautomated system for blood volume analysis has become available. This new system is accurate and straightforward, automatically compares results with appropriate reference values, and can provide results within about $90 \mathrm{~min}$. Also, the last few years have brought increasing recognition of the importance of blood volume abnormalities in the pathophysiology of various conditions, the shortcomings of clinical volume status assessment, and the potential benefit of rapid, accurate volume status assessment. As a result, there has been a surge of interest in blood volume analysis studies.

This article will present a few fundamental concepts of blood volume and circulation physiology, review standard nuclear medicine techniques for analyzing blood volume, give an overview of the new procedure, and introduce some 
of the expanding clinical applications for blood volume analysis information.

\section{OVERVIEW OF BLOOD VOLUME PHYSIOLOGY}

Understanding blood volume analysis requires familiarity with the composition of blood and of some of the physiologic processes involved in circulation and volume homeostasis.

\section{Components of Blood Volume}

For our purposes, blood can be considered to have 2 components: red blood cells (RBCs) and plasma (Fig. 1). Total blood volume (TBV) is the sum of red cell volume (RCV) plus plasma volume (PV). White blood cells and platelets, although extremely important, account for less than $0.1 \%$ of blood volume and, so, can be disregarded. $\mathrm{RBCs}$, the disk-shaped cells responsible for carrying oxygen, compose slightly less than half of blood volume. RBCs are suspended in the plasma, which is a clear, straw-colored fluid. Plasma is about $92 \%$ water plus other solutes, in particular plasma proteins. The most abundant plasma protein is albumin. Plasma proteins serve to transport materials such as hormones, but probably their most important function is to maintain the oncotic pressure of the blood. The term oncotic refers to the force exerted by the plasma protein concentration that draws water into the blood vessels from surrounding tissues through osmosis.

\section{Hematocrit}

If a tube of blood is spun in a centrifuge, the RBCs settle to the bottom of the tube and the plasma remains on top. Hematocrit is the fraction of TBV (expressed as a percentage) that is RBCs. Measured by spinning down a tube of blood and then comparing the height of the column of RBCs with the height of the entire sample (Fig. 1), hematocrit thus gives the volumetric relationship between RBCs and plasma. The reference ranges for hematocrit are $37 \%-41 \%$ in women and $42 \%-47 \%$ in men. Clinically, hematocrit is often used as a measure of the amount of red cells (although this assumes a normal PV, which is not always the case!). On the other hand, because RCV is usually more stable over time than PV, hematocrit is also used clinically as an indicator of PV status. Critical for our purposes, hematocrit is a numeric factor that allows RCV or PV to be calculated once we have measured the other quantity.

\section{Plasma Transudation}

The space within the arteries, veins, and capillaries is termed the vascular compartment of the body. Surprisingly, it is crucial for survival that some of the blood exit from the vascular compartment during circulation. This is because cells require a moist environment to live and also so that substances such as nutrients and wastes carried by the bloodstream can be exchanged with cells some distance from the nearest blood vessel. To accomplish this exchange, a small amount of the plasma (about $0.25 \% / \mathrm{min}$ ), leaks or transudes out of the vascular compartment and forms the interstitial fluid that bathes and nourishes individual cells (Fig. 2). This leakage from vessels occurs predominantly toward the arterial side of capillaries, where some of the plasma water is forced out of the vascular compartment into the interstitial space by arterial pressure (1). On the venous side of capillaries, most of the fluid is drawn back into circulation by osmosis driven by the oncotic pressure exerted by the plasma proteins, which primarily remain in circulation. The remainder of the leaked plasma drains into the lymphatic system, which returns it to the vascular compartment via the thoracic duct in the chest.

\section{Fluid Balance}

Fluid balance in the body is extremely dynamic. Normally, we take in about 2-3 L of fluid daily, mostly in food and beverages, plus the small amount produced by cell
FIGURE 1. Composition of blood and components of blood volume. Cellular components are suspended in plasma. Hematocrit (arrow) is fractional relationship between PV and RCV.

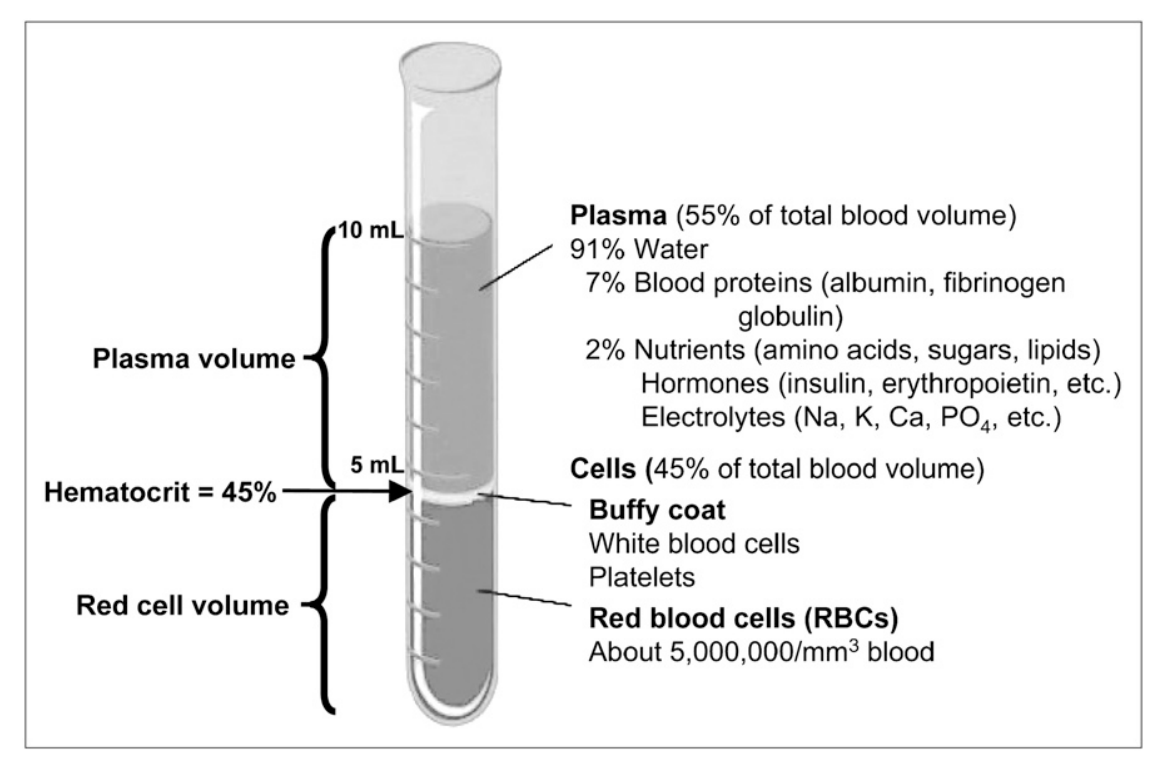




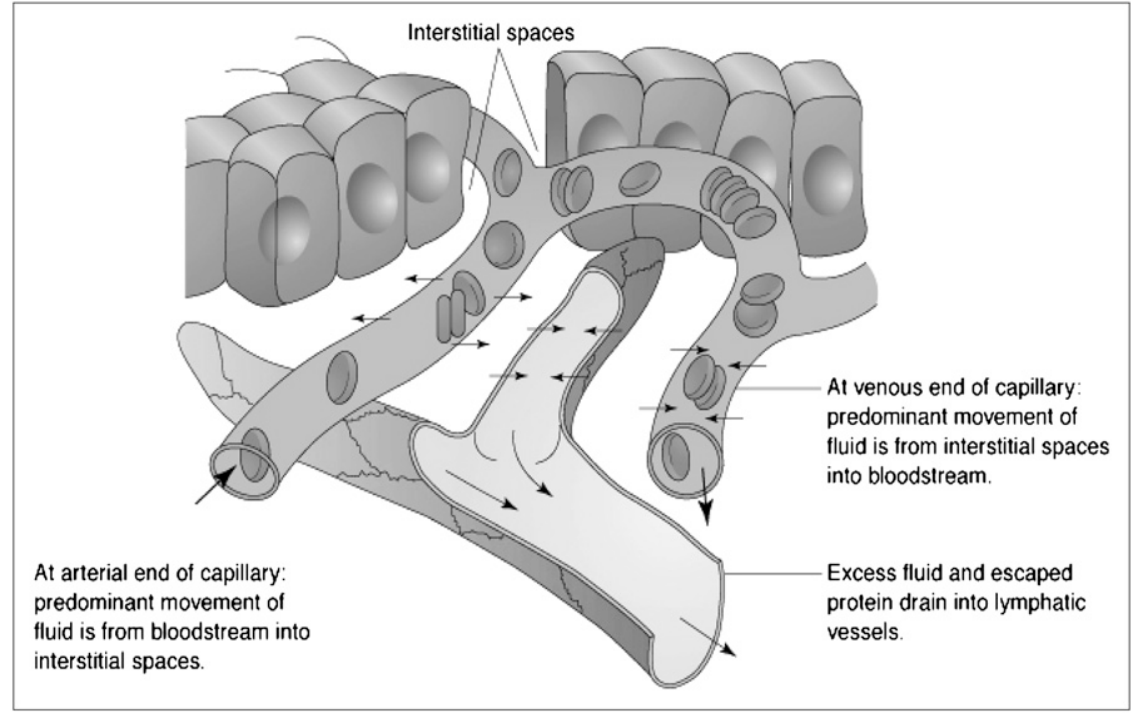

FIGURE 2. Fluid exchanges through capillary membranes in formation and removal of interstitial fluid. (Reprinted with permission of (1).) metabolism. On the other hand, the body loses fluid in urine, feces, respiration, and through the skin (Fig. 3) (2).

In health, fluid input essentially equals fluid output. In illness, however, fluid balance is often severely disrupted. Bleeding, diarrhea, vomiting, burns, surgery, renal failure, dialysis, intravenous fluids, transfusions, and fever all dras-

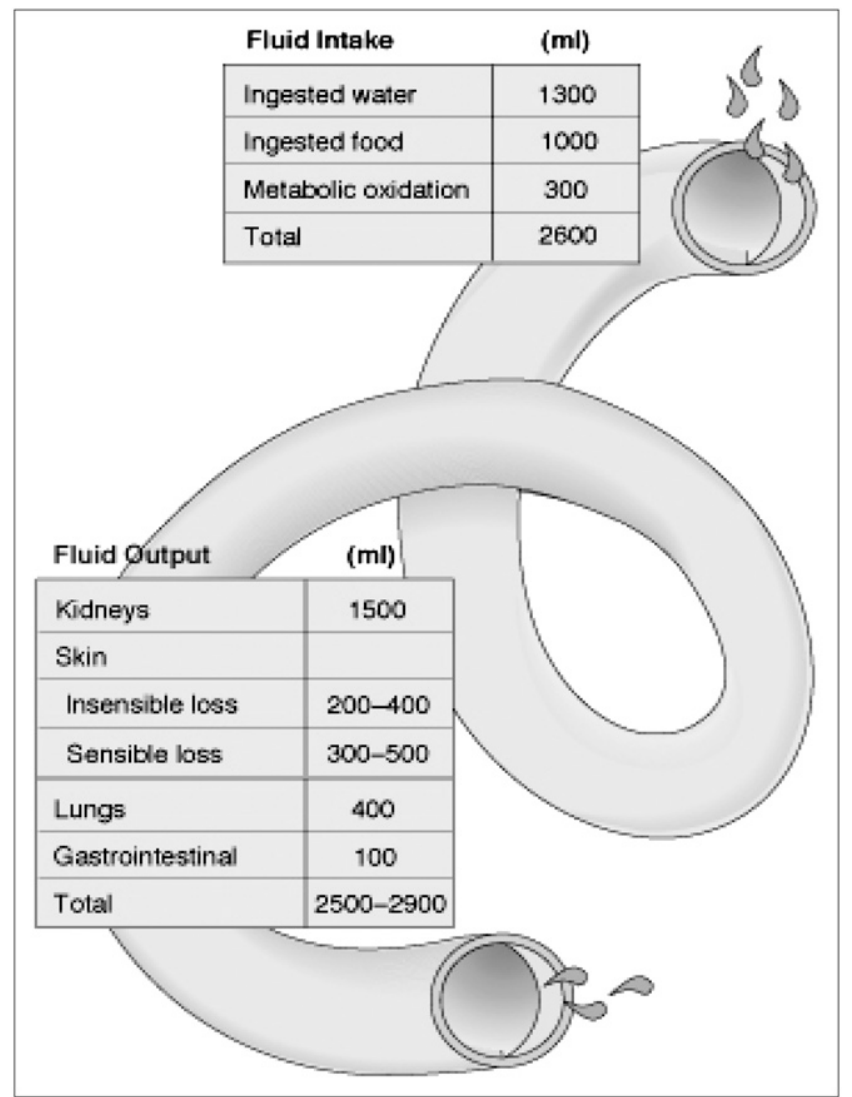

FIGURE 3. Fluid balance. In health, fluid intake and fluid losses are about equal. Amounts shown are average adult daily fluid sources and losses. (Reprinted with permission of (2).) tically affect volume status. Much of the art of patient care, particularly in the critical-care setting, consists of skill in assessing and managing patients' volume status issues. Hypoor hypervolemia, if severe, can lead to potentially fatal complications such as shock, renal failure, or congestive heart failure. Invasive techniques such as pulmonary artery catheterization are sometimes needed to monitor volume status in difficult cases.

\section{BLOOD VOLUME MEASUREMENT AND THE INDICATOR DILUTION PRINCIPLE}

Obviously, we cannot drain the blood to measure it directly. An alternate way to measure an unknown volume is called the indicator dilution technique. This technique refers to the addition of a known volume of a known concentration of some tracer to an unknown volume of liquid. After complete mixing, the concentration of the tracer is measured in a sample taken from the unknown volume. The size of the unknown volume is inversely proportional to the concentration of the tracer in the sample (i.e., the larger the unknown volume, the more diluted the tracer becomes, and the sample therefore contains a lower concentration of tracer.) The unknown volume can then be calculated using the equation $\mathrm{C}_{1} \mathrm{~V}_{1}=\mathrm{C}_{2} \mathrm{~V}_{2}$, where $\mathrm{C}_{1}$ is the concentration of tracer injected, $V_{1}$ is the volume of tracer injected, $C_{2}$ is the concentration of tracer in a sample of the unknown volume, and $\mathrm{V}_{2}$ is the unknown volume.

Figure 4 shows how the indicator dilution technique can be used to measure blood volume. Blood volume has been measured with the indicator dilution technique using dyes such as Evans blue and indocyanine green, but these methods are cumbersome and unreliable. Nuclear medicine is uniquely well suited to perform indicator dilution studies, because our instruments can accurately measure tracer concentrations in fluid samples. In fact, radioisotope blood volume measurements using the indicator dilution technique 
FIGURE 4. How indicator dilution technique is used to measure blood volume. Known quantity of tracer is administered $(A)$, time is allowed for complete mixing $(\mathrm{B})$, and blood sample is drawn for counting (C). Typical nuclear tracers are ${ }^{51} \mathrm{Cr}$-labeled erythrocytes (RBCs) and ${ }^{125} \mathrm{I}-\mathrm{HSA}$.
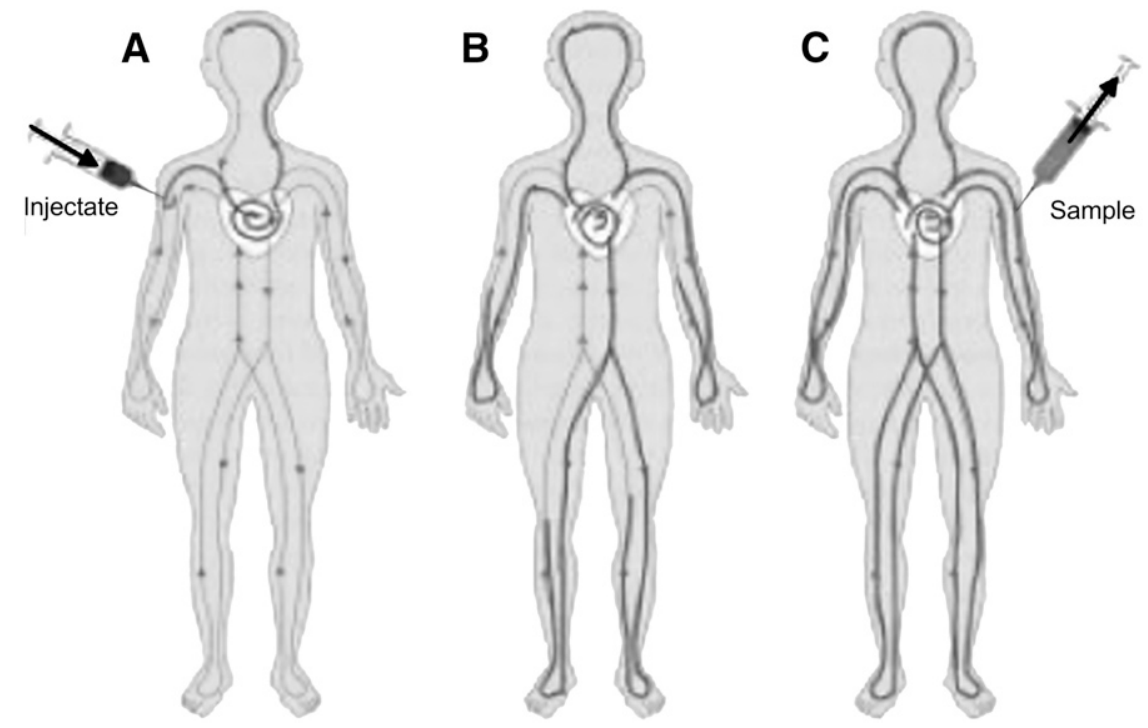

have been used for nearly 60 y (3-6) and have become the gold standard for blood volume assessment.

Both PV and RCV can be measured with radioisotope tracers. To measure PV, human serum albumin (HSA) is tagged with ${ }^{131} \mathrm{I}$ or ${ }^{125} \mathrm{I}$. To measure RCV, the patient's red cells are withdrawn and labeled with ${ }^{51} \mathrm{Cr}$-sodium chromate. For either technique, a standard is prepared with a known quantity of tagged cells or albumin in a known volume. An identical specimen (the injectate) is injected intravenously. After time is allowed for mixing, one or more blood samples are withdrawn. The radioactivity in the blood samples is compared with the radioactivity in the standard to calculate the unknown volume. Because studies with either radiolabeled albumin or ${ }^{51} \mathrm{Cr}$-RBCs yield either only plasma or RCV, the other volume (and the TBV) is calculated using hematocrit.

Over time, the gold standard for blood volume measurement became a dual-isotope/dual-tracer technique involving simultaneous RCV measurement using ${ }^{51} \mathrm{Cr}-\mathrm{RBCs}$ and PV measurement using ${ }^{125}$ I-HSA (7). Although this technique provides extremely accurate results, the double-labeling procedure is long and complex. It requires the preparation of 2 injectates and 2 standards, one of which requires the withdrawal and reinfusion of the patient's own blood. At best, the test requires $4-6 \mathrm{~h}$ or more to complete, and the steps present many opportunities for error. Because of its length and complexity, few nuclear medicine departments actually perform the dual-isotope/dual-tracer technique. (For that matter, departments that have done blood volume analysis studies at all seldom took the time to analyze multiple samples, thereby limiting the accuracy of the studies.) Over the years, among clinicians who were aware of blood volume analysis studies, some developed a perception that the test took too long and that the results were not always reliable. With the exception of hematologists (who use
RBC volume determination in the diagnosis of polycythemia vera), blood volume assessment has for years remained far from the clinical mainstream. This is changing, however, with the arrival of a new technique for analyzing blood volume plus new clinical interest in the results of blood volume analysis.

\section{MAKING THE ANALYSIS USEFUL: WHAT IS THE NORMAL VOLUME?}

In order for an accurately measured blood volume to be meaningful for a particular patient, we need to know what that patient's blood volume should be-that is, we need to know what is normal for that particular patient. Obviously, larger people have larger blood volumes, but blood volume also varies greatly by body composition.

Initial attempts to predict normal blood volume used a fixed ratio of blood volume to body weight, with different ratios for men and for women. However, fat is much less vascular than lean tissue, so 2 individuals of the same weight with different body compositions can have markedly different normal blood volumes. Some early attempts to address this problem included categorizing individuals by body type $(8,9)$, but this method proved too subjective.

Later studies attempted to establish blood volume norms using body surface area as a predictor (10-15), and in 1995 the International Council on Standardization in Hematology published a recommendation for predicting blood volume based on body surface area (16). Compared with body weight, body surface area does more closely estimate normal blood volume but does not adequately reflect the physiologic factors (body mass and the vascularity of different types of tissue) that affect blood volume. Additionally, the International Council on Standardization in Hematology recommended a normal range of $\pm 25 \%$ from predicted, 
which is too wide to be useful for interpreting blood volume in an individual patient.

In 1977, Feldschuh and Enson (17) presented an easily measured, physiologically meaningful method for calculating normal blood volume, based on an estimate of body composition. This method uses the Metropolitan Life height and weight tables to determine an ideal weight at which individuals of different heights have approximately the same proportion of lean tissue to fat. An individual's normal blood volume is predicted on the basis of the percentage that his or her body weight varies from ideal weight. Because an individual below ideal weight is assumed to have a higher proportion of lean tissue, the predicted normal blood volume per unit of mass is higher. Because an individual with a weight above ideal is assumed to have a higher proportion of body fat, the predicted normal blood volume per unit of mass is lower. This method provided a good fit with measured blood volume in 160 healthy individuals with a wide variety of body compositions and avoided the systematic errors that arose from norms based on body weight and body surface area. Using their SD data, Feldschuh and Enson also established a category system for interpreting the severity of blood volume abnormalities, using increments of $\pm 8 \%$. Under that system, a normal blood volume or PV is within $8 \%$ of the predicted normal, mild hypo- or hypervolemia is from $\pm 8 \%$ to $\pm 16 \%$, moderate is from $\pm 16 \%$ to $\pm 24 \%$, severe is from $\pm 24 \%$ to $\pm 32 \%$, and extreme is a more than $32 \%$ deviation from predicted norm. RCV is analyzed in $\pm 10 \%$ increments because it varies more among healthy subjects.

\section{AUTOMATION COMES TO BLOOD VOLUME ANALYSIS}

Recent technologic advances in blood volume assessment have yielded a new method that is simple enough for clinical use and can provide results in approximately 90 min. Approved by the Food and Drug Administration in 1998, the BVA-100 blood volume analyzer (Daxor Corp.) is a semiautomated system for blood volume analysis.

The BVA-100 consists of an automated well counter interfaced with a computer. The instrument is used in conjunction with prepared standards and injectates (Fig. 5). The injectate is a precise amount of ${ }^{131}$ I-labeled HSA (370$1,295 \mathrm{kBq}[10-30 \mu \mathrm{Ci}])$ in saline contained in a patented volumetric flow chamber that ensures essentially complete dose delivery. The BVA-100 uses the indicator dilution principle to measure PV. The hematocrit is then used to calculate the RCV and TBV.

There was initially some controversy about whether using PV measurement in conjunction with hematocrit is as accurate as double labeling (18-21). To settle this question, Dworkin et al. directly compared blood volume analysis results from double labeling with those from the BVA-100 (22). No significant difference was found between the semiautomated results and those from the gold standard test, and the authors concluded that the 2 studies were equiv-
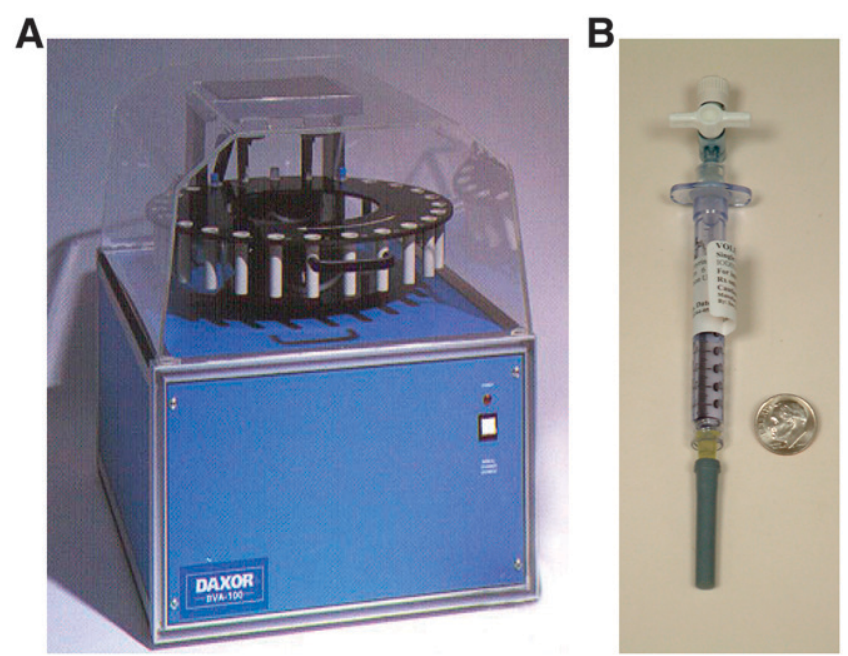

FIGURE 5. (A) Daxor BVA-100 blood volume analyzer. (B) Volumex injectate, containing $370-1,110 \mathrm{kBq}(10-30 \mu \mathrm{Ci})$ of ${ }^{131} \mathrm{I}$-HSA in exactly $1.0 \mathrm{~mL}$ of saline.

alent. Moreover, it took an average of $90 \mathrm{~min}$ for results to be available with the BVA-100, compared with an average of $5 \mathrm{~h}$ with the double-labeling technique.

The new system offers several major advantages over previous nuclear medicine techniques for blood volume analysis. The procedure is less complicated and much more rapid than double labeling and does not involve labeling of the patient's blood. The offsite preparation of the standards and injectates allows for greater precision and quality control. The system uses volume data from 5 sample points instead of just 1 or 2 , and each sample is counted in duplicate, making the results much more reliable. Corrections for mean body hematocrit, trapped plasma, and albumin transudation are incorporated into the system. Finally, the BVA-100 automatically compares each patient's results with his or her predicted normal values based on deviation from ideal weight. This semiautomated system of assessing blood volume and categorizing blood volume derangements has proven to be meaningful in multiple preliminary studies (23-26), and blood volume analysis is increasingly being incorporated into clinical and research practice.

\section{HOW IS THE TEST PERFORMED?}

\section{Sample Collection}

The first steps in performing the study are to accurately measure the patient's height and weight and gain secure intravenous access. After the patient (if ambulatory) has been reclining for several minutes, a control blood sample is drawn. The technologist then administers the injectate by flushing it out of the volumetric delivery chamber and starts a timer. After 12 min (to allow for mixing), blood sampling begins. Five blood samples are drawn into supplied tubes at 6-min intervals. The timing of the samples is actually not critical, as long as the time at which each sample was drawn is accurately recorded. This portion of the test can be 
performed either in the nuclear medicine department or at the bedside.

\section{Sample Processing}

For each sample, duplicate microhematocrit measurements are performed. Each sample is then centrifuged, and two 1-mL aliquots of plasma are removed by pipette. These are placed in the BVA-100 machine along with the standards and tubes for blank and background counts. Counting is performed automatically and takes about $20-40 \mathrm{~min}$, depending on the activity injected.

\section{Data Analysis and Quality Control}

After counting is complete, the preliminary data appear on the screen (Fig. 6). The computer displays the data from the duplicate hematocrit measurements and the counts for the baseline, standard, and duplicate plasma samples. The computer automatically flags duplicate measurements that vary to an unacceptable degree. It also provides an SD measurement that is used to evaluate the validity of the results. If the SD is above 3.8\% after any obvious errors in duplicate measurement have been corrected, the technologist can identify and exclude erroneous or outlying sample points by using the visual display of the regression curve, as described below.

\section{Regression Curve}

The BVA-100 screen includes a graph such as the one in Figure 7. The graph (which is actually a semilogarithmic plot) shows how the instrument arrives at its final results and is important in quality control. The horizontal axis is the time of sampling, whereas the vertical axis reads in TBV $(\mathrm{mL})$. Each point shown on the graph is a separate blood volume determination. Notice that as time increases, the measured volume goes up, reflecting transudation of some of the albumin into the interstitial space (in effect, making volume of distribution for the tracer larger). The computer determines the best-fit line through all the points and then determines the true volume by extrapolating the line back to time 0 .

The use of 5 points facilitates the identification of a single point that is invalid because of random or technical error. With 5 sample points, one can examine the curve, identify if any point is a noticeable outlier, exclude it from the analysis, and still have enough data points to allow an

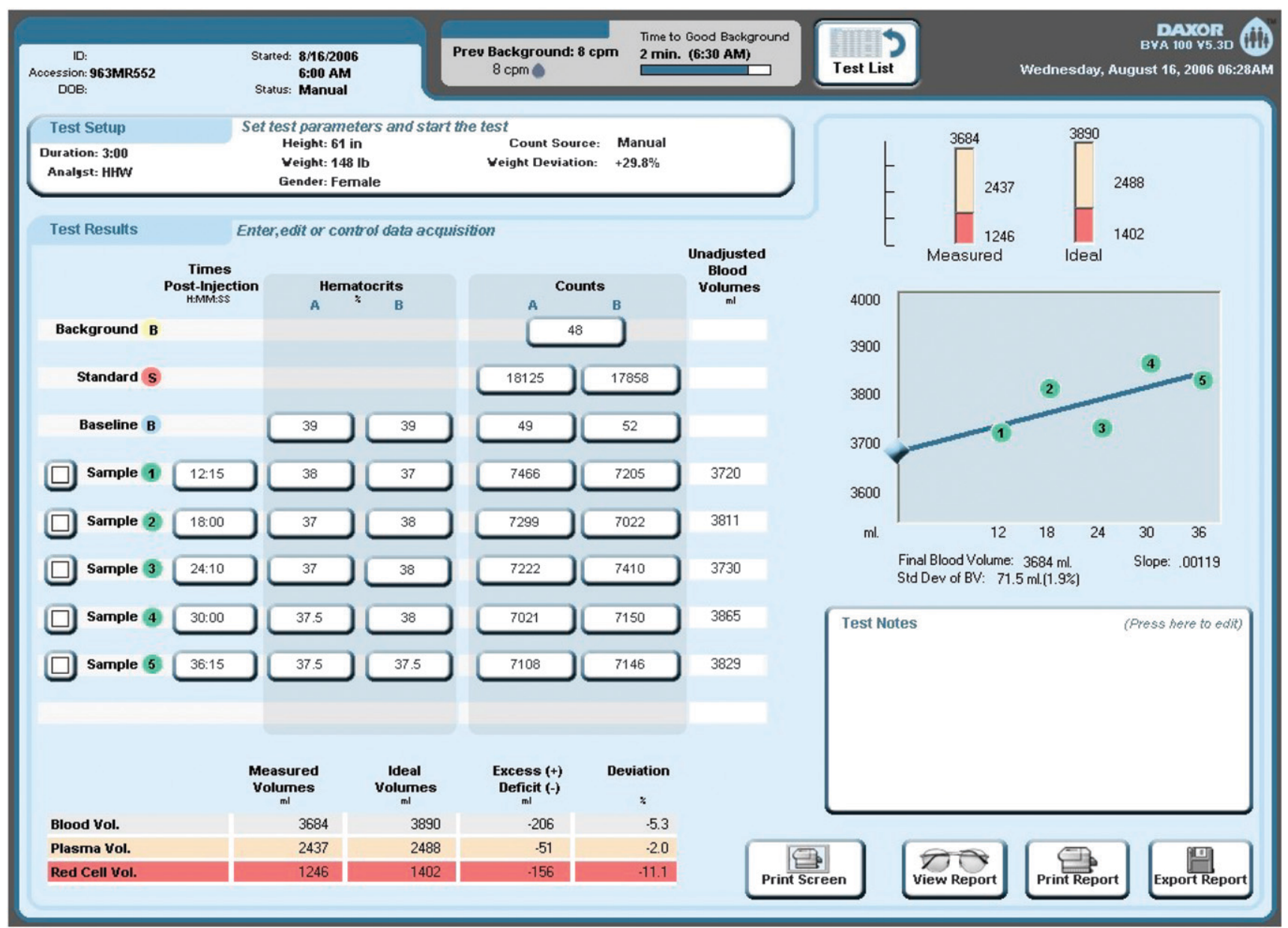

FIGURE 6. BVA-100 results screen, showing sample counts (center), regression line (right), and final patient volume results (bottom) compared with ideal normal values. 


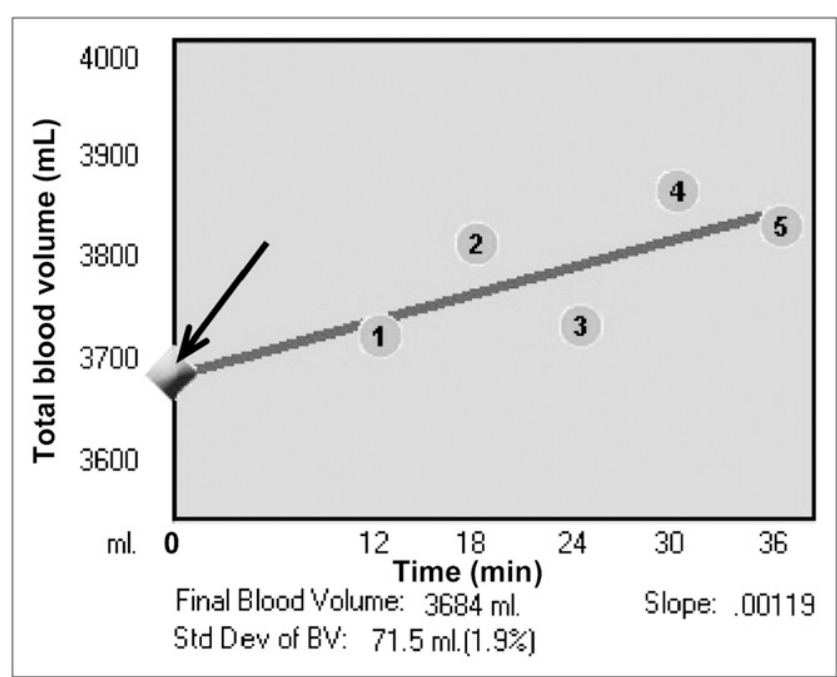

FIGURE 7. Detail of BVA-100 screen showing plot of individual blood volume results for timed samples. Regression line from this plot is extended back to $y$-axis at time 0 (arrow) to give final volume result.

accurate calculation. With fewer points, it is much harder to detect and identify a single erroneous point.

For example, one possible error can occur with the first sample point. When a tracer is injected into the bloodstream, it must mix throughout the entire circulatory system before samples are withdrawn. Although injected tracer will mix with the major circuits of the blood in as little as 1 min, some subcircuits within the cardiovascular system, such as the skin and the muscles at rest, have a much slower circulation time. In a normal patient, mixing should be complete within 8-13 min. In a patient with reduced cardiac output, it may take as long as $20 \mathrm{~min}$ for mixing to be complete (6). If a blood sample is taken before full mixing, the concentration of the tracer will be erroneously high, and the measured blood volume will be erroneously low.

If the SD is high and there are no apparent measurement errors, the technologist can visually identify and remove outlying points on the regression curve. The computer automatically recalculates the SD. Usually the removal of 1 , or possibly 2, erroneous points will result in an acceptable $\mathrm{SD}$ and a valid measurement result.

Occasionally, the SD is high and cannot be corrected through the removal of 1 or even 2 points. In those instances, the result may be valid, but the final interpretation should take into account that the result may include some error. For example, a patient with a high SD and a severe red cell deficit probably has significant anemia. On the other hand, a patient with a high SD and only a borderline red cell deficit may in fact have a normal RCV or may be truly anemic. To facilitate this analysis, the SD is presented both as a percentage and in milliliters, allowing direct comparison of the absolute amount of the patient's volume abnormality and the amount of the SD. If the measured volume derangement is much greater than the $\mathrm{SD}$, then the abnormality is likely a true one. If the volume abnormality is close to the $\mathrm{SD}$, the study is equivocal.

\section{HOW ARE BLOOD VOLUME ANALYSIS RESULTS INTERPRETED?}

The report for a blood volume analysis includes 3 basic parameters: TBV, RCV, and PV. Each parameter is expressed in milliliters and as percentage deviation from the predicted normal value. In addition, the report provides the $\mathrm{SD}$, the normalized hematocrit, and the slope of the line. As we have already seen, the SD gives an indication of the accuracy of the study.

\section{Blood Volume Parameters}

$\mathrm{TBV}, \mathrm{RCV}$, and PV are reported in 2 ways: in milliliters and as percentage deviation from the predicted normal volume. The percentage deviation from normal is considered first, to determine whether the patient has a normal, depleted, or expanded volume. The absolute value in milliliters is important for quantifying the blood volume derangement and making treatment decisions. For example, if a patient requires a transfusion, the blood volume deficit in milliliters can help determine how many units of blood are needed.

When one is interpreting blood volume analysis studies, the results should be examined in this order: TBV first, RCV next, and PV last. In particular, PV should always be examined in relation to RCV and TBV. The normal homeostatic mechanisms of the body are designed to maintain a normal TBV, even when RCV is depleted or expanded. PV is actually abnormal only to the extent to which it fails to maintain a normal TBV.

For example, if a patient has an RCV deficit of $20 \%$, then PV should be expanded so that TBV is normal; this degree of expansion is compensatory. If, however, PV is contracted, normal, or expanded less than necessary to maintain a normal TBV, then that PV is abnormally contracted. If $\mathrm{PV}$ is expanded more than necessary, so that TBV is expanded, then PV expansion is partly compensatory and partly pathologic. Conversely, if RCV is expanded, then PV should contract to maintain a normal TBV. If PV is expanded, then that expansion is entirely pathologic. However, with an expanded RCV, a balance must be maintained between keeping a normal TBV and avoiding excessive hemoconcentration; some TBV expansion may be physiologic in this situation.

In all cases, the patient's blood volume status must be considered in relation to clinical status, comorbid conditions, other measurement results, and medications. There are often several different, possibly opposing, factors contributing to a patient's blood volume disturbance. Particular attention should be paid to the effects of medications (e.g., diuretics or vasoconstrictors) that may directly or indirectly affect volume status. 


\section{Normalized Hematocrit}

Normalized hematocrit provides a single measurement that can be used to evaluate the patient's red cell expansion or depletion in much the same way that peripheral hematocrit has traditionally been used. Basically, normalized hematocrit is a measurement of what the patient's hematocrit would be if the PV were fully expanded or contracted to maintain a normal TBV. Although normalized hematocrit does not provide information that the measured blood volume results do not, it does provide a single measurement that can be used as the hematocrit is often used and that reflects the true degree of red cell derangement.

If the patient is not bleeding, does not receive a transfusion, and is not taking erythropoietin, then peripheral hematocrit actually can be used to track changes in the patient's blood volume for some time after an initial blood volume analysis. This can be done by assuming that the $\mathrm{RCV}$ remains constant and that changes in hematocrit reflect changes in PV. On the other hand, if RCV is known or suspected to be changing, then a follow-up blood volume analysis is needed to evaluate changes in volume status.

\section{The "Slope": A Measure of Capillary Permeability}

As we already know, the BVA-100 calculates the rate of albumin transudation as part of the process of measuring blood volume. The flux of fluids and proteins across the capillary membranes is an important component of blood volume balance and is obviously a fundamental clinical parameter. A high rate of transudation can indicate increased capillary permeability, which may occur in conditions such as burns, sepsis, or rarely capillary leak syndrome. Some clinicians, particularly in critical care, are becoming interested in capillary permeability measurement, although it is not yet clear how this parameter will be used clinically.

On the blood volume analysis report, the rate of transudation is reported as the slope and is presented in units of percentage per minute. A slope of 0.0025 means that albumin is leaving circulation at a rate of $0.25 \% / \mathrm{min}$ Although the normal range for slope has not been fully determined, some studies suggest that normal slope ranges from 0.0005 to $0.0045-0.0050$ (17). In general, when one is evaluating blood volume analysis results, a slope of $0.0045-0.0050$ can be considered equivocal, and a slope of 0.0050 or more may suggest increased capillary permeability. Increased capillary permeability can occur in patients with septic shock or burns and may be present in postoperative, edematous, and hypoalbuminemic patients. It is important to recognize that a patient with abnormal capillary permeability may nonetheless have a normal slope. This possibility can occur when a new balance between intravascular and extravascular albumin has been achieved, particularly in patients with hypoalbuminemia. In addition, when one is evaluating the slope, the technical reliability of the blood volume measurement should be considered. If the SD is low, the slope is more likely to be a reliable indicator of capillary permeability; if the SD is high, the slope may not be as accurate.

Although a normal slope may be as low as 0.0005 , a negative slope suggests a technical error. A negative slope is not a physiologic state, because a negative slope indicates that the concentration of tracer in the plasma is increasing over time.

\section{NEW CLINICAL FRONTIERS FOR BLOOD VOLUME ANALYSIS}

As noted at the beginning of this article, the availability of a semiautomated blood volume analysis system for nuclear medicine departments has brought about a surge in clinical interest in the information that blood volume analysis can provide. There is increasing recognition that blood volume analysis is more accurate than the combination of clinical assessment and surrogate tests commonly used to assess volume status. The literature is starting to suggest new ways for blood volume analysis data to change and improve patient care.

A recent study compared expert clinical assessment of volume status with blood volume analysis results. Experienced cardiologists classified the blood volume status (i.e., hypovolemic, normovolemic, or hypervolemic) of 65 nonedematous, ambulatory heart failure patients (23). Compared with blood volume analysis findings, the clinical assessment was correct only $51 \%$ of the time. Even more compelling, among these heart failure patients, 2-y mortality was $55 \%$ for hypervolemic patients but $0 \%$ for normovolemic and hypovolemic patients. The clear link between volume status and outcome was identifiable only with blood volume measurement. The role of volume status derangement in heart failure continues to be investigated but is believed to play a critical role in the pathophysiology of congestive heart failure.

There is evidence that volume status abnormality may underlie some cases of syncope or the unusual condition "postural orthostatic tachycardia syndrome" (27). At least 1 major medical center is now using blood volume analysis as part of the workup for syncope.

In a study of patients in a surgical intensive care unit (24), among 40 patients whose blood volume was considered difficult to assess, blood volume analysis was performed 86 times. The blood volume analysis study led to a change in treatment $36 \%$ of the time. When the results of blood volume analysis changed treatment, subsequent improvement in oxygenation, renal function, vasopressor use, or cardiac index occurred $42 \%$ of the time. These data suggested that blood volume analysis was more accurate (and certainly blood volume analysis is far less invasive) than pulmonary artery catheterization. Moreover, this study showed that information from blood volume analysis can improve clinical management in the critical care setting. Critical care medicine, surgery, and anesthesia all involve management of difficult volume status issues; the availability 
of rapid blood volume assessment by nuclear medicine departments is of tremendous interest.

\section{CONCLUSION}

Blood volume analysis, using the tried-and-true indicator dilution technique, will be returning to nuclear medicine departments. A new semiautomated system, which can be used by any reasonably equipped department, allows blood volume analysis to be done quickly and with the same accuracy as the gold standard technique. The blood volume analysis reports of today include extraordinarily fundamental clinical parameters we could not previously measure. The data give clinicians a rapid scientific measurement of volume status, for use in cases where clinical estimation is equivocal. The literature on blood volume analysis is only now beginning to emerge but suggests potential application for blood volume analysis studies in the management of various conditions, particularly in hematology, cardiology, surgery, and critical care medicine.

One final caveat about blood volume analysis studies: This is not the same blood volume study that laboratory personnel are used to doing, and they should not think about it in the same way. In our department, we used to joke about "STAT" blood volume studies - the very idea was an oxymoron! Now it is a reality. This technique owes its clinical utility, in large measure, to how quickly the results can be provided, particularly in the critical care setting. Although this technique is not difficult, it can be hard to change the "Oh, it's a blood volume study-I'll count it after lunch" mindset that many nuclear medicine professionals have developed over the years. Laboratories that choose to offer this study need to understand the importance of completing the test and reporting the results accurately and as rapidly as possible. (Teamwork helps tremendously.) Laboratories that make this commitment will be rewarded and will contribute significantly to patient care.

\section{REFERENCES}

1. Prezbindowski KS. Study Guide to Accompany Porth's Essentials of Pathophysiology: Concepts of Altered Health States. 2nd Ed. Philadelphia, PA: Lippincott Williams \& Wilkins; 2005:Figures 23, 24.

2. Taylor C, Lillis C, LeMone P. Fundamentals of Nursing: The Art and Science of Nursing Care. 5th ed. Philadelphia, PA: Lippincott Williams \& Wilkins; 2005: 1434, Figure 46-5.

3. Hevesy G, Zerahn K. Determination of the red corpuscle content. Acta Physiol Scand. 1942;4:376-384.
4. Gray SJ, Sterling K. The tagging of red cells and plasma proteins with radioactive chromium. J Clin Invest. 1950;29:1604-1613.

5. Storaasli JP, Krieger H, Friedell HL, Holden WD. The use of radioactive iodinated plasma protein in the study of blood volume. Surg Gynecol Obstet. 1950;91:458-464.

6. Jaenike JR, Schreiner BF Jr, Waterhouse C. The relative volumes of distribution of $\mathrm{I}^{131}$ tagged albumin and high molecular weight dextran in normal subjects and patients with heart disease. J Lab Clin Med. 1957;49:172-181.

7. International Committee for Standardization in Haematology. Recommended methods for measurement of red-cell and plasma volume. J Nucl Med. 1980;21: 793-800.

8. Gregersen MI, Nickerson JL. Relation of blood volume and cardiac output to body type. J Appl Physiol. 1950;3:329-341.

9. Alexander JK, Dennis EW, Smith WG, Amad KH, Duncan WC, Austin RC. Blood volume, cardiac output, and distribution of systemic blood flow in extreme obesity. Cardiovasc Res Cent Bull. 1962-1963(Winter);1:39-44.

10. Samet P, Fritts HW Jr, Fishman AP, Cournand A. The blood volume in heart disease. Medicine (Baltimore). 1957;36:211-235.

11. Baker RT, Kozoll DD, et al. The use of surface area as a basis for establishing normal blood volume. Surg Gynecol Obstet. 1957;104:183-189.

12. Wennesland R, Brown E, Hopper J Jr, et al. Red cell, plasma and blood volume in healthy men measured by radiochromium (Cr51) cell tagging and hematocrit: influence of age, somatotype and habits of physical activity on the variance after regression of volumes to height and weight combined. J Clin Invest. 1959;38: 1065-1077.

13. Nadler SB, Hidalgo JU, Bloch T. Prediction of blood volume in normal human adults. Surgery. 1962;51:224-232.

14. Retzlaff JA, Tause WN, Kielly JM. Erythrocyte volume, plasma volume and lean body mass in adult men and women. Blood. 1969;33:649-661.

15. Hurley PJ. Red cell and plasma volumes in normal adults. J Nucl Med. 1975; $16: 46-52$.

16. Pearson TC, Guthrie DL, Simpson J, et al. Interpretation of measured red cell mass and plasma volume in adults. Br J Haematol. 1995;89:748-756.

17. Feldschuh J, Enson Y. Prediction of the normal blood volume: relation of blood volume to body habitus. Circulation. 1977;56:605-612.

18. Fairbanks VF, Klee GG, Wiseman GA, et al. Measurement of blood volume and red cell mass: re-examination of ${ }^{51} \mathrm{Cr}$ and ${ }^{125} \mathrm{I}$ methods. Blood Cells $\mathrm{Mol}$ Dis. 1996;22:169-186.

19. Balga I, Solenthaler M, Furlan M. Should whole-body red cell mass be measured or calculated? Blood Cells Mol Dis. 2000;26:25-31.

20. Nielsen S, Rodbro P. Validity of rapid estimation of erythrocyte volume in the diagnosis of polycythemia vera. Eur J Nucl Med. 1989;15:32-37.

21. Olmer M, Berland Y, Purgus R, Schultz G. Determination of blood volume in nephrotic patients. Am J Nephrol. 1989;9:211-214.

22. Dworkin HJ, Premo M, Dees S, et al. Comparison of red cell and whole blood volumes as performed using both chromium-51 tagged red cells and iodine-125 tagged albumin versus using I-131 tagged albumin and extrapolated red cell volume [abstract]. J Nucl Med. 2005;46(suppl):378P-379P.

23. Androne AS, Katz SD, Lund L, et al. Hemodilution is common in patients with advanced heart failure. Circulation. 2003;107:226-229.

24. Biuk-Aghai EN, Yamauchi H, Yu M, et al. Blood volume measurements: impact on fluid management [abstract]. Crit Care Med. 2005;33:A37.

25. Mancini DM, Katz SD, Lang CC, et al. Effect of erythropoietin on exercise capacity in patients with moderate to severe chronic heart failure. Circulation. 2003;107:294-299.

26. Shevde K, Pagala M, Tyagaraj C, et al. Preoperative blood volume deficit influences blood transfusion requirements in females and males undergoing coronary bypass graft surgery. J Clin Anesth. 2002;14:512-517.

27. Raj SR, Biaggioni I, Yamhure PC, et al. Renin-aldosterone paradox and perturbed blood volume regulation underlying postural tachycardia syndrome. Circulation. 2005;111:1574-1582. 